\title{
79. On Products of Consecutive Integers
}

\author{
By Shin-ichi Katayama \\ Department of Mathematics, College of General Education, \\ Tokushima University \\ (Communicated by Shokichi Iyanaga, M. J. A., Dec. 12, 1990)
}

1. Diophantine equations involving products of integers have been investigated by many mathematicians. Among these are Erdös [1], L. J. Mordell [4], but these are the equations in two variables. In this paper, we shall show the following diophantine equation in three variables

$$
x(x+1) y(y+1)=z(z+1)
$$

has infinitely many integer solutions and also show there exists an algorithm for obtaining all the integer solutions of (1).

2. In our previous paper [3], we have obtained the following result. We denote the set of all the integer solutions of a diophantine equation $z^{2}=\left(x^{2}-1\right)\left(y^{2}-1\right)+a(a \in Z)$ by $S_{a}$. Then it is easy to verify that the mappings

$$
\begin{gathered}
\sigma:\left(\begin{array}{l}
x \\
y \\
z
\end{array}\right) \longrightarrow\left(\begin{array}{c}
x \\
x y+z \\
\left(x^{2}-1\right) y+x z
\end{array}\right), \quad \tau:\left(\begin{array}{l}
x \\
y \\
z
\end{array}\right) \longrightarrow\left(\begin{array}{l}
y \\
x \\
z
\end{array}\right), \\
\rho_{1}:\left(\begin{array}{l}
x \\
y \\
z
\end{array}\right) \longrightarrow\left(\begin{array}{c}
-x \\
y \\
z
\end{array}\right), \quad \rho_{2}:\left(\begin{array}{l}
x \\
y \\
z
\end{array}\right) \longrightarrow\left(\begin{array}{c}
x \\
-y \\
z
\end{array}\right), \quad \rho_{3}:\left(\begin{array}{l}
x \\
y \\
z
\end{array}\right) \longrightarrow\left(\begin{array}{c}
x \\
y \\
-z
\end{array}\right)
\end{gathered}
$$

are the permutations of $S_{a}$. $G$ denotes the group $\left\langle\sigma, \tau, \rho_{i}\right\rangle(1 \leqq i \leqq 3)$. We denotes the number of the representatives $\#\left[S_{a} / G\right]$ by $t_{a}$. Then we have obtained the following proposition.

Proposition (cf. [3]). The number $t_{a}$ is finite except in case $a=0$, and $t_{a}$ equals the number of the integer points contained in the set $S_{a} \cap R_{a}$, where

$$
R_{a}=\left\{\left(\begin{array}{l}
x \\
y \\
z
\end{array}\right): 0 \leqq x \leqq y \leqq \sqrt{\left(a+1-x^{2}\right) /(2 x+2)}, 0 \leqq z\right\} \quad \text { in case } a>0,
$$

and

$$
R_{a}=\left\{\left(\begin{array}{l}
x \\
y \\
z
\end{array}\right): 1<x \leqq y, 0 \leqq z, \sqrt{\left(x^{2}-a-1\right) /\left(x^{2}-1\right)} \leqq y \leqq \sqrt{\left(x^{2}-a-1\right) /(2 x-2)}\right\}
$$

in case $a<0$.

For the case $a=4$, we have $t_{a}=2$, that is, $S_{a}=G\left(\begin{array}{l}0 \\ 1 \\ 2\end{array}\right) \cup G\left(\begin{array}{l}1 \\ 1 \\ 2\end{array}\right)$. Con- 
gruence consideration shows $G\left(\begin{array}{l}1 \\ 1 \\ 2\end{array}\right)=\left\{\left(\begin{array}{l}x \\ y \\ z\end{array}\right) \in S_{a}: x \equiv y \equiv 1 \bmod 2\right.$ and $z \equiv 2$ $\bmod 4\}$, which will be denoted by $S^{*}$. We denote by $T$ the set of all the integer solutions of (1). Then the mapping $f:\left(\begin{array}{l}x \\ y \\ z\end{array}\right) \rightarrow\left(\begin{array}{l}2 x+1 \\ 2 y+1 \\ 4 z+2\end{array}\right)$ is a bijection from $T$ to $S^{*}$. We denote $\sigma^{*}=f^{-1} \sigma f, \tau^{*}=f^{-1} \tau f$ and $\rho_{i}^{*}=f^{-1} \rho_{i} f$. Then we have

$$
\begin{aligned}
\sigma^{*}:\left(\begin{array}{l}
x \\
y \\
z
\end{array}\right) & \longrightarrow\left(\begin{array}{c}
x \\
2 x y+x+y+2 z+1 \\
2 x^{2} y+x^{2}+2 x y+2 x z+2 x+z
\end{array}\right), \\
\tau^{*}:\left(\begin{array}{l}
x \\
y \\
z
\end{array}\right) & \longrightarrow\left(\begin{array}{l}
y \\
x \\
z
\end{array}\right), \quad \rho_{1}^{*}:\left(\begin{array}{l}
x \\
y \\
z
\end{array}\right) \longrightarrow\left(\begin{array}{c}
-x-1 \\
y \\
z
\end{array}\right), \\
\rho_{2}^{*}:\left(\begin{array}{l}
x \\
y \\
z
\end{array}\right) & \longrightarrow\left(\begin{array}{c}
x \\
-y-1 \\
z
\end{array}\right) \text { and } \quad \rho_{3}^{*}:\left(\begin{array}{l}
x \\
y \\
z
\end{array}\right) \longrightarrow\left(\begin{array}{c}
x \\
y \\
-z-1
\end{array}\right) .
\end{aligned}
$$

$G^{*}$ denotes the group $\left\langle\sigma^{*}, \tau^{*}, \rho_{i}^{*}\right\rangle(1 \leqq i \leqq 3)$. Then we have $T=G^{*}\left(\begin{array}{l}0 \\ 0 \\ 0\end{array}\right)$, that is, $G^{*}$ acts on $T$ transitively. For example, a solution $\left(\begin{array}{l}x \\ y \\ z\end{array}\right)=\left(\begin{array}{l}1 \\ 2 \\ 3\end{array}\right)$ is obtained by $\left(\begin{array}{l}1 \\ 2 \\ 3\end{array}\right)=\sigma^{*} \tau^{*} \sigma^{*}\left(\begin{array}{l}0 \\ 0 \\ 0\end{array}\right)$. Combining these, we have the following theorem.

Theorem. With the above notation, we have $T=G^{*}\left(\begin{array}{l}0 \\ 0 \\ 0\end{array}\right)$.

\section{References}

[1] P. Erdös: Note on products of consecutive integers. J. London Math. Soc., 14, 194-198 (1939).

[2] K. Kashihara: The diophantine equation $x^{2}-1=\left(y^{2}-1\right)\left(z^{2}-1\right)$. Mem. Anan College of Tech., 26, 119-130 (1990) (in Japanese).

[3] S. Katayama and K. Kashihara: On the structure of the integer solutions of $z^{2}=\left(x^{2}-1\right)\left(y^{2}-1\right)+a$ (to appear in J. Math. Tokushima Univ., 24).

[4] L. J. Mordell: On the integer solutions of $y(y+1)=x(x+1)(x+2)$. Pacific J. Math., 13, 1347-1351 (1963).

[5] — : Diophantine Equations. Academic Press, London, New York (1969). 